\title{
The Use of Corticosteroids for Cardiopulmonary Bypass in Adults
}

\author{
Sophie Perchermeier ${ }^{1} \cdot$ Peter Tassani-Prell $^{1}$
}

Accepted: 18 June 2021 / Published online: 10 August 2021

(C) The Author(s) 2021

\begin{abstract}
Purpose of Review Cardiopulmonary bypass for on-pump cardiac surgery induces a systemic inflammation that may contribute to postoperative major complications. To reduce this inflammatory response in patients undergoing heart surgery, the perioperative use of anti-inflammatory corticosteroids has long been recommended to improve clinical outcomes. However, the efficacy and safety of steroids remain still unclear.

Recent Findings We reviewed recent published literature, including the large clinical trials DECS and SIRS and the two metaanalysis by Dvirnik et al. (2018) and $\mathrm{Ng}$ et al. (2020), on mortality and major postoperative complications, such as myocardial complications, atrial fibrillation, stroke, pulmonary adverse events, length of ICU and hospital stay, renal failure, and infection. Summary The perioperative application of corticosteroids did not improve mortality rates beyond standard care or other secondary outcomes, such as myocardial infarction, stroke, renal failure, and infection. The observed increased risk of myocardial damage in patients receiving corticosteroids in the SIRS trial is mainly related to the author-defined CK-MB threshold as indicator for early myocardial injury. Interestingly, the use of steroids may have some beneficial effects on secondary outcomes: they significantly decreased the risk of respiratory failure and pneumonia and shortened the length of ICU and hospital stay, but the mechanism involved in pulmonary injury is multifactorial and it is difficult to evaluate this result. Patients receiving steroids did not have a decreased incidence of atrial fibrillation shown by the two large trials unlike some previous small sample size trials have demonstrated.
\end{abstract}

Keywords Corticosteroids $\cdot$ Cardiopulmonary bypass $\cdot$ Mortality $\cdot$ Myocardial injury

\section{Introduction}

Cardiopulmonary bypass (CPB) during heart surgeries has been one of the greatest achievements since the 1950s and has become a fundamental part of the modern cardiac surgery. Despite relevant improvements in surgical procedures, anesthesia management, and postoperative care, the risk of major adverse events still persists in patients undergoing heart surgery $[1,2]$. One of the major complications is the systemic inflammatory response to cardiopulmonary bypass [3-5]. The

This article is part of the Topical Collection on Cardiovascular Anesthesia

Sophie Perchermeier

sophie.perchermeier@tum.de

1 Institute of Anesthesiology, German Heart Center, Technical University of Munich, Lazarettstr. 36, D-80636 Munich, Germany exposure to foreign surfaces, non-physiologic blood flow, and hypothermia during CPB induces an inflammatory immune response, including the release of tumor necrosis factor (TNF)- $\alpha$ and interleukin (IL)-6 [6, 7]. This complex inflammatory reaction may be responsible for postoperative complications, such as hypotension, organ dysfunction, and interstitial damage $[8,9]$. Therefore, the perioperative use of corticosteroids, which are well-known to have anti-inflammatory effects, seems reasonable to diminish overshooting immune responses during $\mathrm{CPB}$. Corticosteroids prevent migration of inflammatory immune cells into the circulation, reduce the release of intracellular cytokines, and decrease vascular permeability. However, there is still limited evidence of improving the individual clinical outcome in adults after cardiac surgery. The systematic review and meta-analysis published in 2020 by $\mathrm{Ng}$ et al. could not show a beneficial effect on mortality rates by the perioperative administration of corticosteroids [10•]. These findings support the recommendation of the 
2019 EACTS Guidelines on Cardiopulmonary Bypass in Adult Cardiac Surgery where the routine use of prophylactic corticosteroids is not recommended for adults during cardiac surgery using CPB (Class of Recommendation III, Level of Evidence A) $[11,12]$. Besides their beneficial antiinflammatory effect, corticosteroids can induce hyperglycemia, which impairs wound healing, higher rates on postoperative infections, gastrointestinal bleedings, and renal dysfunction. As a result, corticosteroid administration during cardiac surgery is still under discussion and an analysis of mortality and morbidity is required. A recently published survey by Akhtar et al. showed that $18 \%$ of European and nonEuropean cardiothoracic anesthesiologists still routinely used steroids in their perioperative setting [13]. To clarify the evidence of efficacy and safety, this review will summarize the effects of corticosteroids on mortality, myocardial injury, atrial fibrillation, stroke, pulmonary complications, length of ICU and hospital stay, renal failure, and infections in adults undergoing cardiac surgery.

\section{Mortality and Myocardial Injury}

Pooled data of 16,000 and 14,700 patients, respectively, revealed no significant effect on mortality regarding the administration of perioperative corticosteroids shown by the two recent meta-anaylsis by Dvirnik et al. (2018) and Ng et al. (2020) [10•, 14•]. However, both authors did not exclude the possibility that there may be a beneficial effect of corticosteroids that did not achieve statistical significance $[10 \bullet, 14 \bullet]$. Prior published metaanalysis of corticosteroids reducing mortality rates pointed in that direction but included studies with smaller sample sizes $[15,16]$. The ongoing research and discussion is mainly influenced by two large randomized clinical trials (RCTs): Dexamethasone for Cardiac Surgery (DECS trial) published in 2012 by Dieleman et al. [17••] and Steroids In caRdiac Surgery (SIRS trial) by Whitlock and colleagues in 2015 [18••]. In SIRS, 7507 patients undergoing cardiac surgery from 80 hospitals across 18 countries were randomly assigned to be treated with intravenous methylprednisolone $250 \mathrm{mg}$ at anesthetic induction and $250 \mathrm{mg}$ at the beginning of the cardiopulmonary bypass $(n=3755)$ or placebo $(n=3750)$. The second large trial (DECS) treated 2239 patients with high-dose dexamethasone $(1 \mathrm{mg} / \mathrm{kg}$, max. $100 \mathrm{mg})$ or placebo $(\mathrm{n}=2255)$. Although the mortality rate was higher in patients recruited to the SIRS trial (death within 30 days: 154 steroid (4\%) vs. 177 (5\%) placebo patients) in comparison to the DECS trial $(1.4 \%$ steroid vs. $1.5 \%$ placebo group), no significant benefit from perioperative corticosteroids on mortality rate could be investigated $[17 \bullet \bullet, 18 \bullet \bullet$. The major difference between the two trials are the results on myocardial complications. While the DECS trial showed no differences in the onset of myocardial infarction (1.6\% steroid vs. $1.7 \%$ placebo group), the SIRS trial demonstrated that steroids significantly increased the risk of myocardial injury as part of the composite primary outcome ( $13 \%$ steroids vs. $11 \%$ placebo, $p=0.002$ ). The observed rise of myocardial adverse events in patients receiving steroids might simply be related to biochemical measurements: the SIRS trial differentiated between early perioperative myocardial injury (within $72 \mathrm{~h}$ ) defined as a rise in CK-MB mass or activity, angiographic occlusion of coronary artery, or imaging evidence of new loss of viable myocardium, and late perioperative myocardial injury (later than $72 \mathrm{~h}$ ) with the presence of ECG changes and elevated cardiac markers (troponin or CK$\mathrm{MB})$. These diagnostic criteria were derived from a masked analysis of the first 7000 patients to establish a prognostically relevant postoperative $\mathrm{CK}-\mathrm{MB}$ threshold according to the method developed by Mazumdar and colleagues [19]. However, the DECS trial defined myocardial infarction according to the criteria of the Universal Definition of Myocardial Infarction [20] as a rise of cardiac biomarkers (preferably troponin) together with symptoms of ischemia, ECG changes, imaging of new loss a viable myocard, or new regional wall motion abnormalities. As a matter of fact, since heart surgery is always associated with myocyte damage from the surgical trauma and the period of ischemia despite the use of cardioplegia, the definition of myocardial damage is challenging, but the rise of routinely measured $\mathrm{CK}-\mathrm{MB}$ levels as a sole criterium for early myocardial injury might not be sufficiently specific enough to derive the definition of myocardial injury from it. Indeed, the increased risk of myocardial injury with steroids was only reported in early myocardial events $(<72 \mathrm{~h})$ that were non-Q-wave infarctions (RR 1.24, 1.09-1.41), while the incidence of late myocardial injury $(>72 \mathrm{~h}$ ) did not differ between patients receiving methylprednisolone and placebo, respectively (RR 0.87; 95\% CI 0.49-1.57) [18••]. These results defining myocardial damage as the presence of ECG changes coincide with the findings of the DECS trial that the use of steroids did not increase the risk of myocardial infarction. So far, Whitlock et al. concluded that the DECS trial probably missed some asymptomatic myocardial injuries that where masked by analgesic medications [18••], since only 74 myocardial infarctions were reported $(1.7 \%)$ in the DECS trial versus 885 myocardial injuries in the SIRS trial (12\%), but with consideration of only Q-wave infarctions the distribution with 45 (SIRS trial) and 74 patients (DECS trial) were comparable. Furthermore, the combination of the post-hoc analysis on the incidence of myocardial infarction (defined as the presence of new Q-waves) in the SIRS trial and the sub-analysis of the remaining studies by the meta-analysis of $\mathrm{Ng}$ and coworkers revealed no significant increase in myocardial adverse events between the corticosteroid and placebo group (OR 0.90, 95\% CI 0.70-1.16; participants $=14512$, studies $=23)[10 \bullet]$. The underlying reason for the different outcomes of myocardial adverse events might be related to the used criteria: the SIRS trial defined with the rise of CK-MB levels a myocardial injury, while the DECS trial using 
the Universal Definition of Myocardial Infarction defined a myocardial infarction. The clinical relevance and consequence of the detected increase of myocardial injury by the SIRS trial remain unclear, since the mortality rate is equal between the steroid and the placebo group. Future studies should highlight the mechanism of myocardial damage associated with the use of corticosteroids at the time of cardiac surgery with a precise definition of myocardial infarction and injury.

\section{Atrial Fibrillation and Stroke}

The incidence of postoperative atrial fibrillation ranging from 11 to $35 \%$ is still a frequent and a serious complication after cardiac surgery [21,22], since it is associated with an increased risk for stroke [23] and higher mortality rates [24]. It has previously been hypothesized that the inflammatory response induced by cardiopulmonary bypass and surgical damage causes new atrial fibrillation $[25,26]$. Regarding corticosteroids, there is no clear evidence that the perioperative use significantly reduces the incidence of new atrial fibrillation. The large studies SIRS and DECS, including almost 12,000 patients, did not show a significant difference between the steroid and the placebo group regarding the onset of atrial fibrillation $[17 \bullet \bullet, 18 \bullet \bullet]$. However, the recent meta-analysis of $\mathrm{Ng}$ et al. concluded that steroids reduced the risk of atrial fibrillation (OR $0.87,95 \%$ CI $0.81-0.94$; participants $=14$ 148, studies $=24)$ [10॰]. Interestingly, the authors investigated a treatment-by-dosage interaction of corticosteroids in a subgroup analysis ( $\mathrm{n}=924,6$ trials) [25-29]: favoring low(total dose $<1 \mathrm{~g}$ of hydrocortisone-equivalent) over high-dose (total dose $>1 \mathrm{~g}$ of hydrocortisone-equivalent) corticosteroids ( $\mathrm{p}$ $=0.001)$, i.e., the two large trials using both high-dose corticocosteroids (1 mg/kg dexamethasone and $500 \mathrm{mg}$ prednisolone, respectively) might have dismissed the optimum dosage to achieve beneficial effects on atrial fibrillation. However, since the significant decrease of atrial fibrillation is mainly caused by previous small sample size trials (ranging from 57 to 241 participants) [25-30] with issues regarding double-blinding, randomization, and adequate follow-up and the recently published large trials do not show any differences with steroids $[17 \bullet \bullet, 18 \bullet \cdot]$, we assume that the incidence of new atrial fibrillation might not be altered by the perioperative use of corticosteroids.

In accordance, the incidence of postoperative stroke with $1.3 \%$ versus $1.4 \%$ in the DECS and $2 \%$ in the SIRS trial were not altered by the perioperative use of steroids.

\section{Pulmonary Complications and Length of ICU and Hospital Stay}

Respiratory failure is a severe postoperative complication in patients after cardiac surgery. It has been hypothesized that the perioperative use of corticosteroids may improve pulmonary gas exchange by reducing the increase of inflammatory markers [31]. In both trials (DECS and SIRS), respiratory failure was defined as postoperative mechanical ventilation for an uninterrupted period exceeding $48 \mathrm{~h}$. In the DECS trial, only 67 patients (3.0\%) compared with 97 patients (4.3\%) in the placebo group experienced respiratory failure, indicating that the risk of respiratory failure is significantly reduced by steroids (RR $0.69 ; 95 \%$ CI $0.51-0.94 ; p=0.02$ ). In addition, the perioperative administration of steroids significantly decreased the average time of ventilation from 14.3 to $11.0 \mathrm{~h}(\mathrm{p}<0.001)$ [17••]. These improved pulmonary conditions might cause the earlier discharge from the intensive care unit and a shortened hospital stay which was found by the meta-analysis of Dvirnik et al. and $\mathrm{Ng}$ et al. $\left[10^{\bullet}, 14 \bullet\right]$. However, these positive effects are inconsistent with the results reported by the SIRS trial: the incidence of respiratory failure and stay at the intensive care unit and hospital did not differ between the methylprednisolone and placebo group. Comparable numbers of patients $343(9 \%)$ receiving steroids versus $375(10 \%)$ placebo patients suffered from respiratory failure (RR 0.91) [18••]. Other clinical trials ( $\mathrm{n}=50-125$ patients) with the application of medium dose steroids did not show a significant difference in times of assisted breathing after surgery [32-34], but the postoperative oxygenation index at 12 and $24 \mathrm{~h}$ were significantly improved [34]. In strong contrast to that, Chaney et al. investigated even a significiantly longer period of ventilation time of $2 \mathrm{~h}$ with the perioperative use of steroids (12.8 with steroids vs. $10.1 \mathrm{~h}$ in mean in placebo patients, $\mathrm{p}=0.05$ ) [31]. In addition, in 2009, the meta-analysis by Ho and colleagues demonstrated that high-dose corticosteroids were associated with prolonged intubation of $2.1 \mathrm{~h}(95 \%$ CI 1.76-2.52; $\mathrm{p}<$ 0.01 ) [15]. These opposing results of ventilation times with the use of perioperative corticosteroids in adults undergoing cardiac surgery clearly highlight the importance of future trials. Interestingly, the DECS trial investigated that postoperative pneumonia in the treatment group was reduced from 10.6 to $6 \%$ (RR 0.56; 95\% CI 0.46-0.69; $\mathrm{p}=0.001$ ). This result is particularly exciting, since in a study evaluating the impact of pneumonia, affected patients had a significantly higher hospital mortality rate (28\% with pneumonia vs. $6.2 \%$ matched controls, $\mathrm{p}<0.0001)$ and a higher mortality at the end of follow-up (53\% vs. $19 \%, p<0.0001$ ) [35]. Unfortunately, the data of pneumonia lack in the SIRS trial, since they are not separately shown. $\mathrm{Ng}$ et al. concluded in their recent published meta-analysis that the incidence of pulmonary adverse events were significantly reduced in patients receiving perioperative steroids in comparison to placebo (OR 0.86, 95\% CI 0.75-0.98; participants = 13 426; studies $=17$ studies), but defining pulmonary adverse events as the incidence of pulmonary edema, pleural effusion, pneumonia, pulmonary embolism, or respiratory failure. The effects of perioperative corticosteroids on pulmonary complications are still under discussion and a closer look is needed: Apart from to the use of different steroid doses (low- versus high-dose steroids), the definition of pulmonary adverse events in the clinical trials 
varies between the studies ( $24 \mathrm{~h}$ or $48 \mathrm{~h}$ of uninterrupted ventilation time, including or excluding the incidence of pleural effusion, pulmonary edema, or pneumonia). These different definitions of pulmonary complications have led to the high variance between the results and makes it difficult to compare the data. In addition, intraoperative and postoperative administration of anesthetics and analgetics are not constantly reported, but their use are well-known to play a crucial role in length of ventilation times [36]. The observed clinical benefit of respiratory failure and the reduced incidence of postoperative acquired pneumonia due to the perioperative use of corticosteroids by Dieleman et al. (DECS trial, 2012) is promising [17••], but the mechanism involved in pulmonary injury is multifactorial and makes it difficult to evaluate this result. Alveolar ischemia and hypoxic pulmonary vasoconstriction induced by reduced blood supply during CPB and the surgical trauma (with or without opening the pleura) are only two aspects that need to be considered as additional reasons for respiratory failure [37]. Further studies on respiratory failure with focus on lung compliance, oxygenation index, and ventilation perfusion ratio with strict definitions of pulmonary adverse events and the exact knowledge about sedation during intensive care and the type of surgery might clarify the observed beneficial pulmonary outcomes.

\section{Postoperative Infection}

Since several inflammatory cytokines, including IL-6 and $\mathrm{TNF}-\alpha$, have been measured in high concentrations after CPB $[7,38]$, the use of corticosteroids as anti-inflammatory and immunosuppressive agents seems reasonable to diminish this inflammatory immune response [39]. The clinical trials DECS and SIRS analyzed the incidence of postoperative infections and surgical site infections as part of their secondary study end points. The incidence of surgical site infections was similar in both groups with an event rate from 1.4 (DECS trial) to $4 \%$ (SIRS trial) $[17 \bullet \bullet, 18 \bullet \bullet$. Interestingly, the DECS trial found a significantly reduced incidence of postoperative infection of $9.5 \%$ (vs. $14.8 \%$ ) (RR 0.64; 95\% CI 0.54-0.75; $\mathrm{p}=0.001$ ) in the steroid group, but this effect was primarly related to the reduced incidence of pneumonia in the dexamethasone group ( $6 \%$ vs. $10.6 \%$, $\mathrm{p}<0.001)$. However, the incidence of infection did not differ between the two groups in the SIRS trial $(12 \%$ vs. $13 \%$, RR 0.94). Dvirnik et al. found in their meta-analysis a beneficial effect, since infection occurred in $10.3 \%$ of patients in the steroid group, compared with $12.5 \%$ in the placebo group (RR 0.83; 95\% CI 0.75-0.91; $p<0.0001)$, but the authors discussed their findings as a misinterpretation of the postoperative inflammatory response as an infection. Finally, the other meta-analysis by $\mathrm{Ng}$ et al. reported a lower rate of surgical site infections [10•]. To summarize, although there are some results that corticosteroids can reduce infections, there is no conclusive evidence that they truly have an impact on postoperative infections.

\section{Renal Failure}

Patients undergoing cardiac surgeries are often elderly with multiple comorbidities. It is therefore not suprising that the development of acute kidney injury often occur in patients after cardiac surgeries $[40,41]$. The authors of the DECS trial defined renal failure according to the Risk-Injury-FailureLoss-End stage kidney disease (RIFLE) classification by the Acute Dialysis Quality Initiative [42]. Whitlock et al. defined new renal failure as the development of new stage III acute kidney injury according to the Kidney Disease Improving Global Outcomes (KDIGO) guidelines. There was no significant effect of steroids on the incidence of renal failure in the DECS (1.3\% steroid vs. $1.8 \%$ placebo group) and SIRS trials (both $4 \%$ ) $[17 \bullet \bullet, 18 \bullet \bullet]$, and the recent meta-analyis $[10 \bullet, 14 \bullet]$.

\section{Conclusion and Ongoing Research}

The use of corticosteroids in patients undergoing cardiac surgery with cardiopulmonary bypass has not been shown to improve mortality rates beyond standard care $\left[10^{\bullet}, 14 \bullet\right]$. Regarding the fact that the risk of mortality after cardiac surgery is small $(\sim 4 \%)$, recent meta-anyalsis did not achieve the required population sample size to detect a relative risk reduction of $20 \%$ using two-sided alpha of $5 \%\left[10^{\bullet}, 14 \bullet\right]$. Due to this lack of power, the importance of very large trials rises to give answers regarding therapies with low mortality rates. The observed increase of early myocardial injury (within $72 \mathrm{~h}$ ) by Whitlock and colleagues is interesting, but it is primarily related to the definition of myocardial injury as a rise of CK-MB mass and activity. The data were further attenuated by the result that the incidence of Q-wave myocardial infarction did not differ between the groups [18••], which corresponded with the findings of the DECS trial [17••]. The reduction of pulmonary adverse events along with earlier discharge from the intensive care and hospital may indicate the limited value of mortality as an outcome where the diseasespecific benefit is likely to be in other clinical outcomes [43]. Interestingly, in the DECS trial, patients younger than 65 years had a lower risk for the primary study end point with the administration of steroids (RR 0.65; 95\% CI 0.44-0.96; $\mathrm{p}=0.03$ ). Death, myocardial infarction, stroke, renal, or respiratory failure occurred in 38 patients receiving steroids versus 62 placebo patients. However, patients aged 80 years and older experienced a relative risk of 1.69 to suffer from the primary study end point with the use of corticosteroids (95\% CI $0.92-3.10 ; p=0.09$ ) $[17 \bullet \bullet]$. Therefore, it has been postulated that younger patients suffer from more intense postoperative inflammatory response than elderly individuals after cardiac surgery [17॰•]. The perioperative use of corticosteroids might contribute to the suppression of this inflammatory response and may be especially beneficial for patients aged younger than 65 years [17॰0]. However, such treatment-by-age interaction could not be observed in the SIRS 
trial [18••]. The ongoing DECS-II trial (NCT03002259), a 2800patient, multi-center, randomized trial, has been designed to examine individual outcomes, especially in younger patients (i.e., $<$ 75 years), with a focus on inflammation-related events (such as respiratory failure, kidney injury, sepsis, prolonged ICU, and hospital stay) rather than thrombotic events (myocardial infarction, stroke) and is estimated to be completed in May 2022. Although the two large studies are of high quality, had welldefined clinical outcomes as primary and secondary end points, and longer periods of follow-up ( 6 months), future studies should highlight the impact on mortality and the benefical effect on respiratory complications. As outlined above, a primary challenge will be the definition of myocardial injury and the associated clinical outcome.

Abbreviations CBP, Cardiopulmonary bypass; SIRS trial, Steroids In caRdiac Surgery trial; DECS trial, Dexamethasone for Cardiac Surgery trial; CK-MB, Creatinine kinase-myocardial band; IQR, Interquartile range

Funding Open Access funding enabled and organized by Projekt DEAL.

\section{Declarations}

Conflict of interest The authors declare no commercial or financial conflict of interest.

Open Access This article is licensed under a Creative Commons Attribution 4.0 International License, which permits use, sharing, adaptation, distribution and reproduction in any medium or format, as long as you give appropriate credit to the original author(s) and the source, provide a link to the Creative Commons licence, and indicate if changes were made. The images or other third party material in this article are included in the article's Creative Commons licence, unless indicated otherwise in a credit line to the material. If material is not included in the article's Creative Commons licence and your intended use is not permitted by statutory regulation or exceeds the permitted use, you will need to obtain permission directly from the copyright holder. To view a copy of this licence, visit http://creativecommons.org/licenses/by/4.0/.

\section{References}

Papers of particular interest, published recently, have been highlighted as:

- Of importance

•- Of major importance

1. Mariscalco G, Lorusso R, Dominici C, Renzulli A, Sala A. Acute kidney injury: a relevant complication after cardiac surgery. The Annals of thoracic surgery. 2011;92(4):1539-47.

2. Newman MF, Mathew JP, Grocott HP, Mackensen GB, Monk T, Welsh-Bohmer KA, et al. Central nervous system injury associated with cardiac surgery. Lancet. 2006;368(9536):694-703.

3. Alam SR, Stirrat C, Spath N, Zamvar V, Pessotto R, Dweck MR, et al. Myocardial inflammation, injury and infarction during on- pump coronary artery bypass graft surgery. Journal of cardiothoracic surgery. 2017;12(1):115.

4. Boyle EM Jr, Pohlman TH, Johnson MC, Verrier ED. Endothelial cell injury in cardiovascular surgery: the systemic inflammatory response. The Annals of thoracic surgery. 1997;63(1):277-84.

5. Paparella D, Yau TM, Young E. Cardiopulmonary bypass induced inflammation: pathophysiology and treatment. An update. European journal of cardio-thoracic surgery: official journal of the European Association for Cardio-thoracic Surgery. 2002;21(2): 232-44.

6. Halter J, Steinberg J, Fink G, Lutz C, Picone A, Maybury R, Fedors N, DiRocco J, Lee HM, Nieman G. Evidence of systemic cytokine release in patients undergoing cardiopulmonary bypass. The Journal of extra-corporeal t1 echnology. 2005;37(3):272-77.

7. Roth-Isigkeit A, Borstel TV, Seyfarth M, Schmucker P. Perioperative serum levels of tumour-necrosis-factor alpha (TNFalpha), IL-1 beta, IL-6, IL-10 and soluble IL-2 receptor in patients undergoing cardiac surgery with cardiopulmonary bypass without and with correction for haemodilution. Clinical and experimental immunology. 1999;118(2):242-6.

8. Hennein HA, Ebba H, Rodriguez JL, Merrick SH, Keith FM, Bronstein MH, et al. Relationship of the proinflammatory cytokines to myocardial ischemia and dysfunction after uncomplicated coronary revascularization. The Journal of thoracic and cardiovascular surgery. 1994;108(4):626-35.

9. Sawa Y, Shimazaki Y, Kadoba K, Masai T, Fukuda H, Ohata T, Taniguchi K., Matsuda H. Attenuation of cardiopulmonary bypassderived inflammatory reactions reduces myocardial reperfusion injury in cardiac operations. The Journal of thoracic and cardiovascular surgery. 1996;111(1):29-35.

10. Ng KT, Van Paassen J, Langan C, Sarode DP, Arbous MS, Alston $\mathrm{RP}$, et al. The efficacy and safety of prophylactic corticosteroids for the prevention of adverse outcomes in patients undergoing heart surgery using cardiopulmonary bypass: a systematic review and meta-analysis of randomized controlled trials. European journal of cardio-thoracic surgery : official journal of the European Association for Cardio-thoracic Surgery. 2020;57(4):620-7 This systematic review and meta-analysis updates the reader on the recently published literature. The authors suggested that corticosteroids during cardiac surgeries did not affect mortality rate but reduced pulmonary adverse events.

11. Puis L, Milojevic M, Boer C, De Somer FMJJ, Gudbjartsson T, van den Goor J, et al. 2019 EACTS/EACTA/EBCP guidelines on cardiopulmonary bypass in adult cardiac surgery. Interactive CardioVascular and Thoracic Surgery. 2019;30(2):161-202.

12. Sousa-Uva M, Head SJ, Milojevic M, Collet J-P, Landoni G, Castella M, et al. 2017 EACTS Guidelines on perioperative medication in adult cardiac surgery. European Journal of CardioThoracic Surgery. 2017;53(1):5-33.

13. Akhtar MI, Gautel L, Lomivorotov V, Neto CN, Vives M, El Tahan MR, et al. Multicenter international survey on cardiopulmonary bypass perfusion practices in adult cardiac surgery. Journal of cardiothoracic and vascular anesthesia. 2021;35(4):1115-24.

14. Dvirnik N, Belley-Cote EP, Hanif H, Devereaux PJ, Lamy A, Dieleman JM, et al. Steroids in cardiac surgery: a systematic review and meta-analysis. British journal of anaesthesia. 2018;120(4):65767 This review article summarized the effects of perioperative corticosteroid administration. The authors suggested an unclear impact on mortality and an increased risk of myocardial injury with the perioperative administration of corticosteroids.

15. Ho KM, Tan JA. Benefits and risks of corticosteroid prophylaxis in adult cardiac surgery: a dose-response meta-analysis. Circulation. 2009;119(14):1853-66.

16. Whitlock RP, Chan S, Devereaux PJ, Sun J, Rubens FD, Thorlund $\mathrm{K}$, et al. Clinical benefit of steroid use in patients undergoing 
cardiopulmonary bypass: a meta-analysis of randomized trials. European heart journal. 2008;29(21):2592-600.

17.• Dieleman JM, Nierich AP, Rosseel PM, van der Maaten JM, Hofland J, Diephuis JC, et al. Intraoperative high-dose dexamethasone for cardiac surgery: a randomized controlled trial. JAMA. 2012;308(17):1761-7 The DECS trial published in 2012 is one of two large randomized clinical trials that mainly influences the ongoing research and discussion regarding the use of perioperative corticosteroids in cardiac surgery. The authors summarized that the perioperative administration of dexamethasone was associated with a decreased incidence of postoperative infection, duration of mechanical ventilation, and length of ICU and hospital stay and had beneficial effects on respiratory complications.

18.• Whitlock RP, Devereaux PJ, Teoh KH, Lamy A, Vincent J, Pogue $\mathrm{J}$, et al. Methylprednisolone in patients undergoing cardiopulmonary bypass (SIRS): a randomised, double-blind, placebocontrolled trial. Lancet. 2015;386(10000):1243-53 The SIRS trial published in 2015 is one of the large clinical studies regarding the perioperative use of corticosteroids. The authors concluded that methylprednisolone did not have a significant effect on mortality or major morbidity after cardiac surgery with cardiopulmonary bypass but suggested an increased risk of myocardial injury.

19. Mazumdar M, Smith A, Bacik J. Methods for categorizing a prognostic variable in a multivariable setting. Statistics in medicine. 2003;22(4):559-71.

20. Thygesen K, Alpert JS, White HD, Jaffe AS, Apple FS, Galvani M, et al. Universal definition of myocardial infarction. Circulation. 2007;116(22):2634-53

21. Akintoye E, Sellke F, Marchioli R, Tavazzi L, Mozaffarian D. Factors associated with postoperative atrial fibrillation and other adverse events after cardiac surgery. The Journal of thoracic and cardiovascular surgery. 2018;155(1):242-51.e10.

22. Bhatt HV, Fischer GW. Atrial fibrillation: pathophysiology and therapeutic options. Journal of cardiothoracic and vascular anesthesia. 2015;29(5):1333-40.

23. Wolf PA, Abbott RD, Kannel WB. Atrial fibrillation: a major contributor to stroke in the elderly. The Framingham Study. Archives of internal medicine. 1987;147(9):1561-4.

24. Benjamin EJ, Wolf PA, D'Agostino RB, Silbershatz H, Kannel WB, Levy D. Impact of atrial fibrillation on the risk of death: the Framingham Heart Study. Circulation. 1998;98(10):946-52.

25. Rubens FD, Nathan H, Labow R, Williams KS, Wozny D, Karsh J, et al. Effects of methylprednisolone and a biocompatible copolymer circuit on blood activation during cardiopulmonary bypass. The Annals of thoracic surgery. 2005;79(2):655-65.

26. Vukovic PM, Maravic-Stojkovic VR, Peric MS, Jovic M, Cirkovic MV, Gradinac S, et al. Steroids and statins: an old and a new antiinflammatory strategy compared. Perfusion. 2011;26(1):31-7.

27. Abbaszadeh M, Khan ZH, Mehrani F, Jahanmehr H. Perioperative intravenous corticosteroids reduce incidence of atrial fibrillation following cardiac surgery: a randomized study. Revista brasileira de cirurgia cardiovascular : orgao oficial da Sociedade Brasileira de Cirurgia Cardiovascular. 2012;27(1):18-23.

28. Halonen J, Halonen P, Järvinen O, Taskinen P, Auvinen T, Tarkka $\mathrm{M}$, et al. Corticosteroids for the prevention of atrial fibrillation after cardiac surgery: a randomized controlled trial. JAMA. 2007:297(14):1562-7.

29. Yared JP, Bakri MH, Erzurum SC, Moravec CS, Laskowski DM, Van Wagoner DR, et al. Effect of dexamethasone on atrial fibrillation after cardiac surgery: prospective, randomized, double-blind, placebo-controlled trial. Journal of cardiothoracic and vascular anesthesia. 2007;21(1):68-75.
30. Prasongsukarn K, Abel JG, Jamieson WR, Cheung A, Russell JA, Walley KR, et al. The effects of steroids on the occurrence of postoperative atrial fibrillation after coronary artery bypass grafting surgery: a prospective randomized trial. The Journal of thoracic and cardiovascular surgery. 2005;130(1):93-8.

31. Chaney MA, Nikolov MP, Blakeman B, Bakhos M, Slogoff S. Pulmonary effects of methylprednisolone in patients undergoing coronary artery bypass grafting and early tracheal extubation. Anesthesia and analgesia. 1998;87(1):27-33.

32. Liakopoulos OJ, Schmitto JD, Kazmaier S, Bräuer A, Quintel M, Schoendube FA, et al. Cardiopulmonary and systemic effects of methylprednisolone in patients undergoing cardiac surgery. The Annals of thoracic surgery. 2007;84(1):110-8 discussion 8-9.

33. Oliver WC Jr, Nuttall GA, Orszulak TA, Bamlet WR, Abel MD, Ereth $\mathrm{MH}$, et al. Hemofiltration but not steroids results in earlier tracheal extubation following cardiopulmonary bypass: a prospective, randomized double-blind trial. Anesthesiology. 2004;101(2): 327-39.

34. Yasser Mohamed A, Elmistekawy E, El-Serogy H. Effects of dexamethasone on pulmonary and renal functions in patients undergoing CABG with cardiopulmonary bypass. Seminars in cardiothoracic and vascular anesthesia. 2009;13(4):231-7.

35. Ibañez J, Riera M, Amezaga R, Herrero J, Colomar A, CampilloArtero $\mathrm{C}$, et al. Long-term mortality after pneumonia in cardiac surgery patients: a propensity-matched analysis. Journal of intensive care medicine. 2016;31(1):34-40.

36. Dahan A, Teppema LJ. Influence of anaesthesia and analgesia on the control of breathing. BJA: British Journal of Anaesthesia. 2003;91(1):40-9.

37. Al Jaaly E, Zakkar M, Fiorentino F, Angelini GD. Pulmonary protection strategies in cardiac surgery: are we making any progress? Oxidative medicine and cellular longevity. 2015;2015:416235.

38. Jouan J, Golmard L, Benhamouda N, Durrleman N, Golmard JL, Ceccaldi R, et al. Gene polymorphisms and cytokine plasma levels as predictive factors of complications after cardiopulmonary bypass. The Journal of thoracic and cardiovascular surgery. 2012;144(2):467-73 73.e1-2.

39. Fillinger MP, Rassias AJ, Guyre PM, Sanders JH, Beach M, Pahl J, et al. Glucocorticoid effects on the inflammatory and clinical responses to cardiac surgery. Journal of cardiothoracic and vascular anesthesia. 2002;16(2):163-9.

40. Bastin AJ, Ostermann M, Slack AJ, Diller GP, Finney SJ, Evans TW. Acute kidney injury after cardiac surgery according to risk/injury/failure/loss/end-stage, acute kidney injury network, and kidney disease: improving global outcomes classifications. Journal of critical care. 2013;28(4):389-96.

41. Machado MN, Nakazone MA, Maia LN. Prognostic value of acute kidney injury after cardiac surgery according to kidney disease: improving global outcomes definition and staging (KDIGO) criteria. PLOS ONE. 2014;9(5):e98028.

42. Bellomo R, Ronco C, Kellum JA, Mehta RL, Palevsky P. Acute renal failure-definition, outcome measures, animal models, fluid therapy and information technology needs: the Second International Consensus Conference of the Acute Dialysis Quality Initiative (ADQI) Group. Critical care (London, England). 2004;8(4):R204-12.

43. Prasad V, Lenzer J, Newman DH. Why cancer screening has never been shown to "save lives" - and what we can do about it. Bmj. 2016;352:h6080.

Publisher's Note Springer Nature remains neutral with regard to jurisdictional claims in published maps and institutional affiliations. 